\title{
QUEEN'S
UNIVERSITY
BELFAST
}

\section{Visual attention to food cues in obesity: An eye-tracking study.}

Doolan, K. J., Breslin, G., Hanna, D., Murphy, K., \& Gallagher, A. M. (2014). Visual attention to food cues in obesity: An eye-tracking study. Obesity, 22(12), 2501-2507. https://doi.org/10.1002/oby.20884

\section{Published in: \\ Obesity}

\section{Document Version:}

Peer reviewed version

Queen's University Belfast - Research Portal:

Link to publication record in Queen's University Belfast Research Portal

\section{Publisher rights}

(C) 2014 The Obesity Society

This is the peer reviewed version of the following article: Doolan, K. J., Breslin, G., Hanna, D., Murphy, K. and Gallagher, A. M. (2014),

Visual attention to food cues in obesity: An eye-tracking study. Obesity, 22: 2501-2507, which has been published in final form

athttp://onlinelibrary.wiley.com/doi/10.1002/oby.20884/abstract. This article may be used for non-commercial purposes in accordance with

Wiley Terms and Conditions for Self-Archiving.

\section{General rights}

Copyright for the publications made accessible via the Queen's University Belfast Research Portal is retained by the author(s) and / or other copyright owners and it is a condition of accessing these publications that users recognise and abide by the legal requirements associated with these rights.

\section{Take down policy}

The Research Portal is Queen's institutional repository that provides access to Queen's research output. Every effort has been made to ensure that content in the Research Portal does not infringe any person's rights, or applicable UK laws. If you discover content in the Research Portal that you believe breaches copyright or violates any law, please contact openaccess@qub.ac.uk. 
Authors: Katy Doolan ${ }^{1}$ (BSc Hons), Gavin Breslin² (PhD), Donncha Hanna ${ }^{3}$ (PhD) Kate Murphy

$3 \quad(\mathrm{MSc})^{1}$ and Alison Gallagher ${ }^{1}(\mathrm{PhD})^{*}$

4 Institutions: ${ }^{1}$ Northern Ireland Centre for Food and Health, University of Ulster, Coleraine

5 BT52 1SA, UK ; ${ }^{2}$ Sport and Exercise Science Research Institute, University of Ulster, Jordanstown BT37 OQB, UK and ${ }^{3}$ School of Psychology, Queen's University Belfast, Belfast,

$7 \quad$ BT7 $1 N N$, UK

8 reward

Running title: Attentional bias to visual food cues in obesity

*Corresponding author: Dr Alison Gallagher

Address: Northern Ireland Centre for Food and Health, University of Ulster, Cromore Road, Coleraine, Co. Londonderry, BT52 1SA.

Telephone: +4428701234178

Email: am.gallagher@ulster.ac.uk 
Objective: Based on the theory of incentive sensitization, the aim of this study was to investigate differences in attentional processing of food-related visual cues between normal weight and overweight/obese males and females.

Design and Methods: Twenty-six normal weight (14M, 12F) and twenty-six overweight/obese (14M, 12F) adults completed a visual probe task and eye-tracking paradigm. Reaction times and eye-movements to food and control images were collected during both a fasted and fed condition in a counterbalanced design.

Results: Participants had greater visual attention towards high energy dense food images compared to low energy dense food images regardless of hunger condition. This was most pronounced in overweight/obese males who had significantly greater maintained attention towards high energy dense food images as compared to their normal weight counterparts however no between weight group differences were observed for female participants. 
- Research has demonstrated that exposure to food visual cues and the subsequent activation of reward pathways in the brain may play a role in obesity.

- To date, a number of researchers have investigated attentional responses to food cues in both normal weight and overweight/obese populations however results have been conflicting.

- Despite eye-tracking being considered to be one of the most direct methods of assessing attentional bias, studies that have been conducted to date using this technique have only addressed differences in the attentional processing of visual food cues between normal weight and overweight/obese individuals within female populations.

\section{What this study adds}

- Results from the present study indicate that HED food images are more 'attention grabbing' than LED food images.

- Findings also provide for the first time, evidence for a possible dysregulation of a visual food cue-associated reward system in overweight/obese males

- This study highlights the need for future studies using eye-tracking as a direct method of assessing visual attention to clarify the potential role of attentional bias may have in the development and maintenance of obesity 


\section{Introduction}

It has been suggested that the current 'obesogenic' environment is contributing greatly to the worldwide obesity epidemic ${ }^{1,2}$. This environment consists of palatable, energy dense foods that are easily accessible and extensively marketed ${ }^{3}$. Research has demonstrated that exposure to high energy dense (HED) food cues and the subsequent activation of reward pathways in the brain may play a role in both the development and maintenance of obesity $^{4,5}$. This concept stems from the theory of incentive sensitization ${ }^{6}$ which suggests that modification of the dopaminergic reward systems in the brain results in increased salience to related visual cues. As attentional bias to food cues is a potentially modifiable factor $^{7-10}$, it is of interest to further understand the potential role enhanced attention to food visual stimuli may have on eating behaviours and food choices as part of an integrated approach to addressing obesity.

To date, a number of researchers have investigated attentional responses to food cues in normal weight ${ }^{11-13}$ and overweight/obese populations $s^{4,14-18}$ however results have been conflicting. For example, a study conducted by Nijs et al. ${ }^{14}$ using a modified Stroop test, observed greater interference to food words in obese participants with higher levels of selfreported food cravings as compared to their normal weight counterparts. In contrast Loeber et al. ${ }^{15}$ used a visual probe task and found no difference in attentional bias to food cues between normal weight and obese individuals. Such inconsistencies within attentional processing research may be explained by the use of different methodological approaches with previous studies using indirect methods, such as the modified Stroop test and visual probe task that do not allow for the assessment of the direction of attentional bias ${ }^{16}$. More 
recently, research has focused on the use of the more direct method using eye-tracking to measure visual attention that overcomes the methodological issues associated with indirect measures of attentional bias ${ }^{19,20}$.

Eye-tracking is a non-invasive method of measuring visual gaze that provides a direct, ecologically valid assessment of attentional bias ${ }^{21,22}$. To date, this technique has been employed in studies investigating attentional processing of visual food cues between normal weight and overweight/obese females. Castellanos et al. ${ }^{4}$ combined eye-tracking with a visual probe task to establish differences in attentional bias to food images in normal weight and obese women in a fasted and fed condition. Both weight groups had increased visual gaze towards food images compared to non-food images in the fasted state. In the satiated condition, obese participants retained incentive salience to food cues. More recently Werthmann et al. ${ }^{16}$ investigated differences in attentional bias for HED related food cues in overweight females compared to normal weight females in a fed state. This study included a visual probe task and recording of eye-movements and observed that overweight/obese females initially directed attention towards food images compared to non-food images to a greater extent than normal weight individuals but found no significant difference in maintained visual attention

Despite eye-tracking being considered as a direct method of assessing attentional bias ${ }^{21,22}$, studies conducted to date using this technique have only addressed differences in attentional processing of visual food cues between normal weight and overweight/obese individuals within female populations ${ }^{4,16,17,18}$. Male participants have been excluded from these studies due to reported gender differences in eating style traits, ${ }^{18,23}$ behavioural and 
neural responses to foods ${ }^{24,25}$ or to improve homogeneity ${ }^{4,17}$ therefore it is unclear whether there are differences in attentional processing of visual food cues as directly assessed by visual gaze between normal weight and overweight/obese individuals in a male population.

Several authors have reported that the energy content of visual food cues may play a role in increased attention to food stimuli ${ }^{4,16,17}$. In line with the theory of incentive sensitization, these studies have suggested HED foods are considered to be more rewarding and as a result HED food visual cues become more salient and receive greater selective attention. HED foods are overtly represented within the visual environment through food advertising ${ }^{16}$ therefore it is of interest to further understand if certain individuals, in particular those who are overweight/obese, have increased attention towards HED food stimuli.

The aim of this study was to extend previous research by investigating the impact of weight status, satiation state and energy density content of food images on attentional processing of visual food cues in females. For the first time, differences in attentional processing of visual food stimuli between normal weight and overweight/obese males using a direct assessment of visual gaze were examined. It was hypothesised that fasting would increase attention to food cues and that overweight/obese individuals would maintain increased attentional bias for food cues when satiated. Furthermore it was hypothesised that participants would have greater attention to HED food images as compared to low energy density (LED) related food images. 


\section{Methods and procedures}

Participants

Participants were recruited through email and poster advertisements. The flow of participants through the study protocol is presented in Figure 1. Eligible participants were healthy males and females aged 18-65 years (mean 29.2 SD 10.7 years), with a body mass index (BMI) of $18.5-34.9 \mathrm{~kg} / \mathrm{m}^{2}$. Participants with a BMI between $18.5-24.9 \mathrm{~kg} / \mathrm{m}^{2}$ were classified as normal weight and those with a BMI of $25-34.9 \mathrm{~kg} / \mathrm{m}^{2}$ were classified as overweight/obese ${ }^{26}$. Exclusion criteria (adapted from Nijs et al. ${ }^{18}$ ) were applied as follows: spectacle wearers or presence of known ocular disease; tobacco users; taking any medication that may influence eating behaviour; presence of any known chronic diseases and participation within the past three months in an intervention aimed at losing weight. Written informed consent was obtained from all participants.

\section{Study design}

The study design was modified from Castellanos et al. ${ }^{4}$. Participants attended on two study days at least 5 days apart and completed the experimental task once in a fasted state and once in a fed state. Participants were instructed to fast for a minimum of 8 hours before both sessions and were randomly assigned to perform the experimental task in the fasted or fed state at the first study session to prevent study order bias. Participants were contacted before their study session to remind them to fast and on the day of the study session, participants were asked by a researcher if they had adhered to this instruction with all participants confirming they had. Participants' height was measured to the nearest millimetre $(\mathrm{mm})$ using a free standing stadiometer and weight measured to the nearest kilogram (kg) using a digital scale. For the fasted trial, participants completed a visual 
analogue scale (VAS) questionnaire ${ }^{27}$ and then immediately undertook the experimental task. The VAS questionnaire was completed to determine participants' self-reported feelings of hunger and fullness. The scale was an anchored line of $100 \mathrm{~mm}$, with for example in question one, $0 \mathrm{~mm}$ equaling "Not at all hungry" and 100mm equaling "As hungry as I have ever felt". For the fed trial, participants consumed a liquid meal (milkshake: 0.65kcal/ml, $61 \%$ carbohydrate, $21 \%$ protein, $18 \%$ fat) provided in an opaque glass, consumed through a straw until further refills were declined and participants reported they could consume no more. The volume of liquid meal consumed was recorded to the nearest millilitre and hunger levels were assessed using VAS approximately twenty minutes after consumption.

\section{Experimental Task}

\section{Visual Probe Task}

Visual stimuli consisted of pairs of images containing 20 LED related foods and non-food matches and 20 HED related foods and non-food matches ${ }^{4}$. HED related food images were considered as those high in fat and/or sugar (e.g. chocolate, pizza ), and LED related food images were considered as those with a high water content and low in fat and/or sugar (e.g. fruits, vegetables). Each food image was matched to a non-food image for size, complexity and colour ensuring it was only the content (whether the stimuli was a food or non-food) that differed between image pairs. Non-food images included items such as stationary and tools.

The experimental task began with a central fixation cross shown for $1000 \mathrm{~ms}$, followed by the image pairs for $2000 \mathrm{~ms}$. After each image pair, a dot probe replaced one of the previous images, remaining until the participant made a manual response by pressing the 
corresponding key on a computer keyboard. Participants were instructed to respond to the probe as quickly as possible. Each image pair was shown twice in a random order with filler images randomly interspersed to reduce monotony. Reaction time data from the visual probe task was collected using E-prime software 2.0. Consistent with previous studies ${ }^{4,11,21}$, incorrect responses, reaction times of less than $200 \mathrm{~ms}$ or greater than $1500 \mathrm{~ms}$ and reaction times exceeding the mean individual reaction time of the participant plus or minus 3 standard deviations were excluded from subsequent analysis. Reaction time bias scores were calculated by subtracting reaction times of congruent trials (probe replaced the food image) from reaction times of the incongruent trials (probe replaced the non-food image). Positive values indicate attention bias towards food images; negative values indicate attention bias away from food images and towards non-food (control) images ${ }^{11}$.

\section{Eye-Movement Data}

A head-mounted eye-tracker was used to collect eye-movement data during the visual probe task. Prior to the beginning of the task, participant eye-movements were calibrated using a 9-point calibration frame. Gaze fixation measurements were sampled every $16 \mathrm{~ms}^{4}$. Fixations were considered as (a) saccades that remained stable for $\geq 100 \mathrm{~ms}^{18}$ (b) the initial fixation was initiated at least $100 \mathrm{~ms}$ after image onset, as fixations $<100 \mathrm{~ms}$ may reflect anticipatory eye-movements ${ }^{18}$ and (c) fixations that were directed to the left or right image $^{15}$. Eye-movement data was analysed using ASL Gaze Tracker software. Gaze fixations that occurred outside of image pairs (e.g. on blank screen/not on the screen) were excluded from data analysis. Two measures were obtained from gaze fixation data; gaze direction bias and gaze duration bias ${ }^{4,18}$. Gaze direction bias is considered as a measure of initial attentional orientation, calculated using the number of trials in which the first fixation was 
directed to a food image as a proportion of all trials in which the fixation was made towards either the food or control image. A direction bias score $>0.5$ indicates attentional bias towards food images; equal to 0.5 represents no bias and $<0.5$ reflects an orienting bias towards control images. Gaze duration bias is considered as a measure of maintained attention, calculated using the average gaze duration to a food image across all trials as a proportion of the average gaze duration to all images (food and control). Similarly to gaze direction bias scores, a duration bias score of $>0.5,0.5$ or $<0.5$ represents maintained attention to food pictures, no bias and maintained attention to control images respectively.

\section{Statistical analyses}

Statistical analyses were conducted using the Statistical Package for the Social Sciences software version 21. All data was considered to be normally distributed. Statistical analyses were conducted firstly at a group level comparing normal weight vs. overweight/obese participants and secondly, due to reported gender differences in eating style traits ${ }^{18}$, data was split by gender for comparison between weight groups in males and females. Independent $t$-tests were used to compare demographic characteristics (e.g. age) between BMI categories. Self-reported hunger levels (measured using VAS) before and after liquid meal consumption were compared using a mixed between-within analysis of variance (ANOVA) with VAS score from the fasted trial and fed trial as within-subject variables and weight group (normal weight vs. overweight/obese) as the between subject variable. Analyses of reaction time and eye-movement data were also conducted using mixed-design ANOVA with weight group as the between-subject factor and hunger condition (fasted vs. fed), image type (food image vs. control image) and food image energy density content (HED food image vs. LED food image) as within-subject factors. One sample $t$-tests were 
used to compare reaction time bias scores to a test value of zero and food image direction and duration bias scores to a test value of 0.5 . An alpha level $p<0.05$ was considered to represent statistical significance throughout.

\section{Results}

\section{Baseline characteristics}

Participant demographics are summarised in Table 1. Both weight $(t(50)=-5.75, p<0.001)$ and BMI $(t(50)=-9.82, p<0.001)$ were significantly different between the study groups (normal weight vs. overweight/obese). Participants in the overweight/obese group were significantly older compared to those in the normal weight group (mean 33.5 SD 12.9 years vs. mean 24.9 , SD 5.2 years respectively; $t(50)=-3.15, p=0.003$ ). There was a main effect of satiety condition, $(F(1,50)=108.8, p<0.001)$ with all participants reporting lower levels of hunger following liquid meal consumption (mean subjective hunger ratings, were 60.3 (SD 21.5) $\mathrm{mm}$ when fed and 17.4 (SD 19.2) $\mathrm{mm}$ when fasted). There was no significant difference in hunger levels or amount of liquid meal consumed between BMI groups ( $F(1$, 50) $=0.025, p=0.874)$

\section{Reaction time bias data}

Mean reaction times and mean reaction time bias scores are given in Table 2 . No significant effects of BMI group or satiety condition were observed for reaction time bias scores. There was a significant main effect for energy density in reaction time bias scores $(F(1,50)=5.15$, $p=0.028$ ) with all participants in a satiated condition having a greater attentional bias towards HED food images (mean reaction time bias score 6.7, SD 43.6) as compared to LED food images (mean reaction time bias score -11.5 , SD 43.5) but this was not observed whilst 
participants were in a fasted condition $(p>0.05)$. One sample $t$-test analysis demonstrated however that reaction time bias scores for HED and LED food images when satiated were not significantly different from a test score of zero $(t(51)=1.1, p=0.063$ and $t(51)=-1.9$, $p=0.275$ respectively)

\section{Gaze direction bias}

Gaze direction bias scores are presented in Table 3. There was a significant main effect of energy density $(F(1,50)=14.64, p<0.001)$ with all participants regardless of satiety condition or BMI group demonstrating greater bias towards HED (mean 0.524, SD 0.05) as compared to LED food images (mean 0.476, SD 0.05). One sample $t$-test analysis demonstrated that participant direction bias scores for HED and LED food images were significantly different from a test score of $0.5(t(51)=3.9, p<0.001$ and $t(51)=-3.8, p<0.001$ respectively).

\section{Gaze duration bias}

A main effect for energy density (Table 3$)$ was observed in gaze duration bias scores $(F(1$, $50)=14.44, p<0.001$ ) with all participants regardless of satiety condition or BMI group attending to HED food images for a longer duration than LED food images (mean gaze duration bias scores of 0.515 (SD 0.05) and 0.485 (SD 0.05) respectively). One sample $t$-tests were conducted for HED and LED food duration bias scores and results demonstrated a significant difference from a test score of $0.5(t(51)=2.2, p=0.04$ and $t(51)=-2.2, p<0.04$ respectively).
Commented [KD2]: Can I state that the results from the ANOVA were significant but when followed up with a one sample $t$ test the values did not significantly differ from a score of zero? 
Males

In terms of gaze direction bias scores, there was a significant main effect of energy density $(\mathrm{F}(1,26)=9.53, p=0.005)$ with all males, regardless of $\mathrm{BMI}$ group or satiety condition, demonstrating greater orienting bias towards HED (mean 0.526, SD 0.05) as compared to LED food images (mean 0.473, SD 0.05).

All male participants demonstrated a significantly greater gaze duration bias towards HED (mean 0.513, SD 0.053) as compared to LED food images (mean 0.491, SD 0.06), $F(1,26)=$ 7.39, $p=0.012$. There was also a statistically significant interaction between BMI group and energy density $(F(1,26)=4.94, p=0.035$; see Figure $2 \mathrm{a})$ with overweight/obese males having a greater gaze duration bias towards HED food images (mean 0.523, SD 0.06) compared to normal weight males (mean 0.502, SD 0.06). No effects for satiety condition were observed in gaze duration bias scores.

\section{Females}

In terms of gaze direction bias scores, female participants had a significantly greater orienting bias towards HED food images (mean 0.521, SD 0.04) as compared to LED food images (mean 0.479, SD 0.05), $F(1,22)=4.87, p=0.038)$.

Analysis of mean gaze duration bias scores in female participants demonstrated a significant main effect for energy density. All females demonstrated a greater gaze duration bias towards HED (mean 0.518, SD 0.05) compared to LED food images (mean 0.477, SD 0.05, $F$ $(1,22)=2.86, p=0.006$; see Figure $2 b)$. 


\section{Discussion}

The results from eye-movement data demonstrate that all participants had greater visual attention to HED food images compared to LED related food images indicating increased attentional bias towards HED visual food stimuli. These results support previous research demonstrating that HED food images are more 'attention-grabbing' than LED food images ${ }^{4}$. HED foods tend to be higher in fat and sugar that have been linked to greater stimulation of reward pathways in the brain which may account for increased attentional bias towards these visual stimuli ${ }^{25,28}$

Previous studies have failed to consider potential weight differences in the attentional processing of visual food cues in a male population ${ }^{4,13,17,18}$. Results from the current study demonstrated that overweight/obese males had significantly greater maintained attentional bias to HED food images compared to normal weight males. These findings are the first to investigate and identify differences in attention to food stimuli using eye-tracking as a direct assessment of visual attention between weight groups in adult males. Findings indicate that greater attention to HED food cues may have a role in the development and maintenance of obesity not only in females as demonstrated by previous research ${ }^{4}$, but also in males. Future studies investigating attentional processing of food visual cues should therefore include males and females.

In contrast to what was hypothesised, no differences between weight groups were observed in regards to visual attention to food images in female participants however there appeared 
to be a trend for overweight/obese females to have reduced attention to HED food images as compared to their normal weight counterparts. The current results are in contrast to the work of Castellanos et al. ${ }^{4}$ who reported that obese females had greater attention to food images compared to normal weight females as measured by eye-tracking data. Other researchers however have suggested that females, in particular overweight females, may employ cognitive strategies to reduce attentional allocation to visual food cues as a means of preventing disinhibited food intake ${ }^{16,18}$. This attentional 'avoidance strategy' may in part explain findings in the current study and highlights the importance of avoiding a 'one size fits all approach' to applying conclusions drawn from investigating attentional processing in obese females to those who are overweight.

No effect for satiety was observed in any attentional processing measures in the present study. This was in contrast to the hypothesis that attention to visual food cues would be moderated by satiety condition and evidence presented in previous studies that demonstrated an increase in attention to food images ${ }^{4}$ and food words ${ }^{29}$ in a fasted condition. One reason for the inconsistency in findings may have been the use of a liquid meal to induce satiety. Although all participants confirmed they had adhered to instructions to fast prior to both study sessions and reported significantly reduced feelings of hunger following consumption of the liquid meal, it is possible participants may have been less hungry than they indicated on visual analogue scales prior to and following study meal consumption 
Reaction time data from the visual probe task did not yield any between-weight group

differences. These results are similar to those observed by Castellanos et $a{ }^{4}{ }^{4}$ who failed to observe any statistically significant differences between weight groups (normal weight versus obese women) in reaction time data from a visual probe task however did report a main effect of weight group in attentional bias scores obtained from eye-tracking data. These results may in part be explained by issues in interpreting results obtained from a visual probe task. Reaction time data is usually considered to be an indirect measure of attention allocation at stimuli presentation offset however it has been suggested that participants may 'shift' their attention from one stimulus to another in tasks with longer stimuli presentation times e.g. $>500 \mathrm{~ms}^{30}$. It may be useful for future studies to employ the recording of eye-movements as a more direct method to provide information on attentional engagement and disengagement to visual food-related cues.

The present study had some limitations that should be taken into consideration. Firstly, although results from the VAS questionnaire suggested participants felt full following consumption of the liquid meal, it may not have been fully effective in inducing satiety. Secondly both overweight and obese individuals were included, perhaps future studies could better account for BMI differences by recruiting equal numbers of normal weight, overweight and obese individuals to allow for direct comparison of potential differences between BMI categories and attention to visual food cues.

Despite these limitations, the present study has several strengths. The use of both direct (eye-tracking) and indirect (visual probe task) methods were used to assess attentional bias 
allowing for direction and duration of initial and maintained visual attention to be measured. As previously discussed, it has been suggested using a direct method or a combination of a direct and indirect method may overcome some of the methodological issues encountered using an indirect measure alone such as the interpretation of the direction of allocated attention ${ }^{31}$. The inclusion of male participants was a novel aspect of this research and to the author's knowledge is the first study to date to identify greater attentional bias to HED food images using a direct method of assessment in overweight/obese males as compared to their normal weight counterparts. Finally, the inclusion of both HED and LED food cues allowed for comparison of energy density content of food images which to date has only been investigated in a limited number of studies using eye-tracking as a direct measure of visual attention in an overweight/obese population $^{4,17}$.

Results from the present study indicate that HED food images are more 'attention grabbing' than LED food images. The findings also provide, for the first time, evidence for a possible dysregulation of a visual food cue-associated reward system in overweight/obese males, with this weight group displaying greater attentional bias towards HED food images than their normal weight counterparts. Future studies using eye-tracking as a direct method of assessing visual attention are required to clarify the potential role of attentional bias in the development and maintenance of obesity.

Conflicts of interest: The authors declare no conflicts of interest 
06

Funding: PhD sponsorship was obtained from the Department for Employment and Learning, Northern Ireland. Eye tracking equipment was funded by the Department for Employment and Learning Research Capital Investment Fund.

Acknowledgements: The authors would like to acknowledge Dr Sinead Smyth for the design of the visual probe task and extend their thanks to those who took part in the study.

Author contributions: $\mathrm{AMG}$ and GB designed the study and KJD recruited participants and conducted the research. KM assisted with participant recruitment, data collection and entry. DH advised on statistical analyses. KJD analysed the data and prepared the first draft of the manuscript. AMG, GB and DH critically reviewed the manuscript and approved the final version. AMG led the research and finalised the manuscript. 


\section{References}

1. Swinburn BA, Sacks G, Hall KD, McPherson K, Finegood DT, Moodie ML et al. The global obesity pandemic: shaped by global drivers and local environments. Lancet 2011; 378: 804814.

2. Foresight. Tackling obesities: future choices - project report ( $2 \mathrm{nd} \mathrm{Ed).} \mathrm{London:} \mathrm{Foresight}$ Programme of the Government Office for Science, 2007. Available at: www.bis.gov.uk/assets/bispartners/foresight/docs/obesity/17.pdf (Accessed 22 ${ }^{\text {nd }}$ August 2013).

3. Blundell JE, Stubbs RJ, Golding C, Croden F, Alam R, Whybrow S et al. Resistance and susceptibility to weight gain: Individual variability in response to a high-fat diet. Physiol Behav 2005; 86: 614-622

4. Castellanos EH, Charboneau E, Dietrich MS, Park S, Bradley BP, Mogg K et al. Obese adults have visual attention bias for food cue images: evidence for altered reward system function. Int J Obes 2009; 33: 1063-1073.

5. Volkow ND, Wise RA. How can drug addiction help us understand obesity? Nat Neurosci 2005; 8: 555-560. 
6. Robinson T, Berridge K. The Neural Basis of Drug Craving - an Incentive-Sensitization Theory of Addiction. Brain Res Rev 1993; 18: 247-291.

7. Smith E, Rieger E. The effect of attentional training on body dissatisfaction and dietary restriction. Eur Eat Disorders Rev 2009; 17: 169-176.

8. Hardman CA, Rogers PJ, Etchells KA, Houstoun, Munafo MR. The effects of food attentional bias training on appetite and food intake. Appetite 2013; 71: 295-300.

9. Kemps E, Tiggemann M, Orr J, Grear J. Attentional retraining can reduce chocolate consumption. J Exp Psychol 2014; 20: 94-102.

10. Kakoschke N, Kemps E, Tiggemann M. Attentiaonal bias modification encourages healthy eating. Eat Behav 2014; 15: 120-124.

11. Mogg K, Bradley B, Hyare H, Lee S. Selective attention to food stimuli in hunger: are attentional biases specific to emotional and psychopathological states, or are they also found in normal drive states? Behav Res Ther 1998; 36: 227-237.

12. Leland D, Pineda J. Effects of food stimuli on visual spatial attention in fasting and nonfasting normal subjects: Behavior and electrophysiology. Clin Neurophysiol 2006; 117: 67-84. 
13. Hepworth R, Mogg K, Brignell C, Bradley BP. Negative mood increases selective attention to food cues and subjective appetite. Appetite 2010; 54: 134-142.

14. Nijs IMT, Franken IHA, Muris P. Food-related Stroop interference in obese and normalweight individuals: Behavioural and electrophysiological indices. Eating Behaviours 2010; 11: 258-265.

15. Loeber S, Grosshans M, Korucuoglu O, Vollmert C, Vollstaedt-Klein S, Schneider S et al. Impairment of inhibitory control in response to food-associated cues and attentional bias of obese participants and normal-weight controls. Int J Obes 2012; 36: 1334-1339.

16. Werthmann J, Roefs A, Nederkoorn C, Mogg K, Bradley BP, Jansen A. Can(not) Take my Eyes off it: Attention Bias for Food in Overweight Participants. Health Psychol 2011; 30: 561-569.

17. Graham R, Hoover A, Ceballos NA, Komogortsev O. Body mass index moderates gaze orienting biases and pupil diameter to high and low calorie food images. Appetite 2011; 56: $577-586$.

18. Nijs IMT, Muris P, Euser AS, Franken IHA. Differences in attention to food and food intake between overweight/obese and normal-weight females under conditions of hunger and satiety. Appetite 2010; 54: 243-254. 
19. Field M, Cox WM. Attentional bias in addictive behaviors: A review of its development, causes, and consequences. Drug Alcohol Depend 2008; 97: 1-20.

20. Duc AH, Bays P, Husain M. Eye-movements as a probe of attention. Prog Brain Res 2008; 171: 403-411.

21. Mogg K, Bradley BP, Field M, De Houwer J. Eye-movements to smoking-related pictures in smokers: Relationship between attentional biases and implicit and explicit measures of stimulus valence. Addiction 2003; 98: 825- 836 .

22. Baschnagel JS. Using mobile eye-tracking to assess attention to smoking cues in a naturalized environment. Addict Behav 2013; 38: 2837-2840.

23. Burton $\mathrm{P}, \mathrm{Smit} \mathrm{HJ}$, Lightowler $\mathrm{HJ}$. The influence of restrained and external eating patterns on overeating. Appetite 2007; 49: 191-197

24. Cornier MA, Salzberg AK, Endly DC, Bessesen DH, Tregellas JR. Sex-based differences in the behavioral and neuronal responses to food. Physiol Behav 2010; 99: 538-543 
25. Geliebter A, Pantazatos SP, McOuatt H, Puma L, Gibson CD, Atalayer D. Sex-based fMRI differences in obese humans in response to high vs. low energy food cues. Behav Brain Res 2013; 243: 91-96

26. World Health Organisation. Obesity and overweight. 2013; Available at: http://www.who.int/mediacentre/factsheets/fs311/en/ (Accessed 22 ${ }^{\text {nd }}$ August 2013.)

27. Hill AJ, Blundell JE. Nutrients and behaviour: research strategies for the investigation of taste characteristics, food preferences, hunger sensations and eating patterns in man. J Psychiatr Res 1983; 17: 203-212.

28. Wang GJ, Volkow ND, Telang F, Jayne M, Ma Y, Pradhan K et al. Evidence of gender differences in the ability to inhibit brain activation elicited by food stimulation. Proc Natl Acad Sci 2009; 106: 1249-54.

29. Lavy EH, Vandenhout MA. Attentional Bias for Appetitive Cues - Effects of Fasting in Normal Subjects. Behav Cog Psychotherapy 1993; 21: 297-310.

30. Field M, Cox W.M. Attentional bias in addictive behaviours: A review of its development, causes and consequences. Drug Alcohol Depen 2008; 97:1-20 
31. Mogg K, Field M, Bradley BP. Attentional and approach biases for smoking cues in smokers: an investigation of competing theoretical views of addiction. Psychopharmacology 2005; 180: 333-341. 


\section{List of tables}

551 Table 1. Baseline characteristics and subjective hunger rating scores of study participants.

552

Table 2 Mean reaction time measures for normal weight and overweight/obese participants in fasted and fed conditions.

555

Table 3 Mean attention bias measures of normal weight and overweight/obese participants in fasted and fed conditions.

558 
Figure 1 Study protocol and participant flow through study.

570

Figure 2 Mean gaze duration bias scores as a function of weight group (normal weight vs.

overweight/obese) and food image energy density content (high energy density vs. low energy density) for (a) males and (b) females.

574

575 
Table 1. Baseline characteristics and subjective hunger rating scores of study participants.

\begin{tabular}{ccc} 
Normal weight & Overweight/obese & $P$ \\
$(n 26)$ & $(n 26)$ \\
\hline Mean & SD & Mean $\quad$ SD
\end{tabular}

\section{Baseline}

characteristics $^{\mathrm{a}}$

$\begin{array}{lccccc}\text { Age (years) } & 24.9 & 5.2 & 33.5 & 12.9 & 0.003 \\ \text { Height }(\mathrm{cm}) & 173.5 & 11.2 & 173.3 & 9.2 & 0.949 \\ \text { Weight }(\mathrm{kg}) & 67.6 & 12.0 & 88.3 & 13.9 & <0.001 \\ \text { BMI(kg/m2) } & 22.2 & 1.9 & 29.3 & 3.2 & <0.001 \\ \text { Study meal (kJ) } & 2319.1 & 302.3 & 2600.3 & 340.0 & 0.454\end{array}$

Subjective hunger rating (VAS) ${ }^{b}$

587 
597 Table 2 Mean reaction time measures for normal weight and overweight/obese participants in 598 fasted and fed conditions.

\begin{tabular}{|c|c|c|c|c|}
\hline & $\begin{array}{c}\text { Normal weight } \\
\text { (n26) }\end{array}$ & \multicolumn{3}{|c|}{$\begin{array}{l}\text { Overweight/obese } \\
\text { (n26) }\end{array}$} \\
\hline$R T s$ (ms) & Mean & SD & Mean & SD \\
\hline Fed HED incongruent & 541.5 & 71.9 & 537.8 & 91.6 \\
\hline Fed HED congruent & 531.5 & 47.9 & 534.4 & 83.5 \\
\hline Fed LED incongruent & 524.5 & 59.5 & 535.3 & 84.9 \\
\hline Fed LED congruent & 541.2 & 70.8 & 541.6 & 79.6 \\
\hline Fasted HED incongruent & 553.9 & 116.2 & 546.7 & 89.0 \\
\hline Fasted HED congruent & 539.6 & 94.3 & 556.7 & 95.2 \\
\hline Fasted LED incongruent & 540.2 & 107.3 & 545.6 & 85.0 \\
\hline Fasted LED congruent & 538.9 & 105.9 & 552.1 & 91.3 \\
\hline RT bias score & & & & \\
\hline Fed HED & 9.9 & 48.9 & 3.4 & 37.9 \\
\hline Fed LED & -16.7 & 49.0 & -6.3 & 37.6 \\
\hline Fasted HED & 14.3 & 44.5 & -10.0 & 36.2 \\
\hline Fasted LED & 1.2 & 56.2 & -6.5 & 55.4 \\
\hline
\end{tabular}

\section{9}

60 Abbreviations: RT, reaction time; HED, high energy density; LED, low energy density.

60 Visual probe task: Incongruent - probe replaces control image, congruent - probe replaces food image.

60 


\begin{tabular}{|c|c|c|c|c|}
\hline & \multicolumn{2}{|c|}{$\begin{array}{l}\text { Normal Weight } \\
\qquad(n 26)\end{array}$} & \multicolumn{2}{|c|}{$\begin{array}{c}\text { Overweight/obese } \\
\text { (n26) }\end{array}$} \\
\hline & Mean & SD & Mean & SD \\
\hline \multicolumn{5}{|l|}{ Gaze direction bias } \\
\hline Fed HED & 0.513 & 0.06 & 0.522 & 0.07 \\
\hline Fed LED & 0.487 & 0.06 & 0.477 & 0.07 \\
\hline Fasted HED & 0.525 & 0.06 & 0.533 & 0.05 \\
\hline Fasted LED & 0.477 & 0.06 & 0.464 & 0.05 \\
\hline \multicolumn{5}{|c|}{ Gaze duration time (ms) } \\
\hline Fed HED food & 441.2 & 136.3 & 450.0 & 120.4 \\
\hline Fed HED control & 419.1 & 117.5 & 431.2 & 93.1 \\
\hline Fed LED food & 424.7 & 135.9 & 392.2 & 71.9 \\
\hline Fed LED control & 430.6 & 114.6 & 438.8 & 109.9 \\
\hline Fasted HED food & 415.3 & 153.5 & 433.3 & 143.9 \\
\hline Fasted HED control & 371.4 & 134.7 & 413.1 & 110.9 \\
\hline Fasted LED food & 391.1 & 166.3 & 405.6 & 112.6 \\
\hline Fasted LED control & 357.2 & 117.6 & 415.9 & 114.5 \\
\hline \multicolumn{5}{|l|}{ Gaze duration bias } \\
\hline Fed HED & 0.504 & 0.08 & 0.519 & 0.07 \\
\hline Fed LED & 0.477 & 0.07 & 0.478 & 0.06 \\
\hline Fasted HED & 0.525 & 0.08 & 0.512 & 0.05 \\
\hline Fasted LED & 0.489 & 0.11 & 0.495 & 0.04 \\
\hline
\end{tabular}

Abbreviations: HED, high energy density; LED, low energy density. 
Figure 1 Study protocol and participant flow through study.

Abbreviations: VAS, visual analogue scale. *Participants were required to fast for a minimum of 8 hours before each trial day and randomly assigned to trial order on first visit. +Eye tracking paradigm - combination of visual probe task and eye tracking. 
Figure 2 Mean gaze duration bias scores as a function of weight group (normal weight vs.

overweight/obese) and food image energy density content (high energy density vs. low energy density) for (a) males and (b) females.

Gaze duration bias score $>0.5,0.5$ or $<0.5$ represents maintained attention to food related images, no bias and maintained attention to non-food (control pictures) related images respectively. Mixed design ANOVA: weight group (normal weight vs. overweight/obese) as between-subject factor; gaze duration bias scores (high energy density vs. low energy density) as within-subject factor. Different letters represent statistically significant differences. 